Advancements in Instrumentation

\title{
Variable-Pitch Dispensing Workstation and Its Application to the Preparation of Microsensor Arrays
}

\author{
Hiroshi AOKI, ${ }^{* \dagger}$ Takashi IKEDA, ${ }^{* *}$ Masaki Torimura,* and Hiroaki TAO* \\ *National Institute of Advanced Industrial Science and Technology (AIST), \\ 16-1 Onogawa, Tsukuba, Ibaraki 305-8569, Japan \\ **C.A.N. Inc., 2517-22 Ajiki, Tsukuba, Ibaraki 305-4205, Japan
}

\begin{abstract}
We have developed an 8-ch capillary-based dispensing workstation with a variable capillary pitch mechanism. The capillary intervals can be varied from 1 to $9 \mathrm{~mm}$ to dispense different solutions simultaneously at an arbitrary dispensing pitch, allowing direct dispensing from microplates to integrated analytical systems. To evaluate the precision of its dispensing performance, droplets of Rhodamine $\mathrm{G}$ dye were dispensed onto glass slides and the values of the optical volume were analyzed. The error in the dispensed volume proved to be $0.54 \mathrm{~nL}$ when dispensing $20 \mathrm{~nL}$. In dispensing small volumes, the volume error for this workstation was found to be about 100-fold less than that seen in conventional dispensers. Even highly viscous solutions containing $50 \%$ glycerol could be dispensed with precision. Rapid dispensing was also achieved. Moreover, the application of the workstation to preparing addressable $8 \times 12$ microsensor array chips was demonstrated, providing an independent and reproducible spot array.
\end{abstract}

(Received April 8, 2008; Accepted April 25, 2008; Published June 10, 2008)

In the past couple of decades, great efforts have been focused on developing techniques for high-throughput analysis of biologically relevant materials, particularly drug discovery in the pharmaceutical industry. ${ }^{1,2}$ These analytical techniques have begun to be applied to clinical assays in mass screenings, e.g., mass spectrometry, ${ }^{3,4}$ DNA microarrays, ${ }^{5}$ protein arrays, ${ }^{6}$ and cell arrays. $^{7-9}$ Techniques for the pretreatment of specimens have also been successfully developed to permit rapid and continuous sample handling. ${ }^{10}$ To manage hundreds of samples a day destined for high-throughput analysis, lab automation has been utilized to perform processes that used to be done manually, from sample preparation to assay. Hitherto, automated sample-handling systems have been designed for precise and effective dispensing with the aim of using SBS standard microplates (or microtiter plates). SBS standards for the design of microplates were proposed by the Society for Biomolecular Screening (SBS) and approved as universal industry standards by the American National Standards Institute (ANSI), to promote cost-effective screening (ANSI/SBS 1-2004 - ANSI/SBS 4-2004).

However, techniques for the introduction of prepared sample solutions to analytical processes which are intermediate between pretreatment and analysis are open to improvement. This is because conventional SBS standard-based dispensing systems are not suitable for dispensing at much higher densities than SBS-based patterns. Even if the analytical techniques were adaptable to integrated analytical systems allowing the measurement of high-density samples, there is no alternative to conventional dispensing systems, resulting in low-efficiency analysis. Moreover, existing dispensing systems are not capable of the simultaneous positioning and integration of biologically

$\dagger$ To whom correspondence should be addressed.

E-mail: aoki-h@aist.go.jp relevant materials including nucleic acids, peptides, and proteins. Furthermore, due to their small sample volume and labile character, these materials need rapid and high-precision dispensing systems.

To meet these requirements, we developed in this study an 8-ch capillary-based dispensing workstation with widely adjustable capillary intervals. ${ }^{11,12}$ Capillaries were placed at even intervals so that the dispensing pitch could be expanded or contracted in the range from 1 to $9 \mathrm{~mm}$. This mechanism allows sample solutions to be dispensed directly from SBS-based microplates to integrated analytical systems and rapid and simultaneous dispensing of up to 8 different solutions. To the best of our knowledge, these cannot be realized using conventional commercially available dispensers. The precision of our dispensing workstation was evaluated by dispensing droplets of Rhodamine $\mathrm{G}$ dye on glass slides. The error in the dispensed volume was found to be $0.54 \mathrm{~nL}$ when dispensing droplets of $20 \mathrm{~nL}$. This error is about two orders magnitude less than that seen in conventional dispensers that use disposable pipette tips in dispensing $1 \mu \mathrm{L}$ of droplets, being so far the smallest practical volumes for pipette dispensing. The droplets were not mixed with adjacent droplets even at a dispensing pitch of $1 \mathrm{~mm}$. It was also found that a highly viscous solution containing $50 \%$ glycerol could be dispensed with precision and that the dispensing was 4.4-8.7-fold faster compared to conventional dispensers. We have thus achieved the development of a high-precision and rapid dispensing workstation.

This workstation was then applied to the preparation of addressable $8 \times 12$ microsensor array chips. These were made from photolithographically fabricated microelectrode array chips by dispensing droplets of 11-hydroxy-1-undecanethiol (11-HUT) and 8-ferrocene-1-octanethiol (8-FcOT) for surface modification. The redox peaks were observed only for the 8 -FcOT modified microelectrodes. For the 11-HUT modified 
electrodes, in contrast, only charging currents were observed, where these two kinds of microelectrodes were separated by $1 \mathrm{~mm}$. This result provides evidence for the reproducible performance of the workstation and confirms that the droplets do not mix.

\section{Experimental}

\section{Reagents}

11-Hydroxy-1-undecanethiol (11-HUT) and 8-ferrocene-1octanethiol (8-FcOT) were purchased from Dojin Laboratory (Kumamoto, Japan). OMR 83, OMR Developer, and Remover 502A were purchased from Tokyo Ohka Kogyo (Tokyo, Japan). SU-8 3000 and SU-8 Developer were purchased from Kayaku Microchem (Tokyo, Japan). Rhodamine G, glycerol, ethanol, 2propanol, $\mathrm{H}_{2} \mathrm{SO}_{4}, \mathrm{H}_{2} \mathrm{O}_{2}, \mathrm{NH}_{4} \mathrm{OH}, \mathrm{KI}, \mathrm{I}_{2}, \mathrm{HClO}_{4}, \mathrm{Ce}\left(\mathrm{NH}_{4}\right)_{2}\left(\mathrm{NO}_{3}\right)_{6}$, $\mathrm{NaClO}_{4}$, and $\mathrm{NaH}_{2} \mathrm{PO}_{4}$ were purchased from Wako Pure Chemicals (Osaka, Japan). An anisotropic conductive film (ACF, ANISOLM) was a gift from Hitachi Chemicals (Tokyo, Japan). All of the chemicals used were of analytical reagent grade. All aqueous solutions were prepared with deionized and charcoal-treated water (specific resistance $>18.2 \mathrm{M} \Omega \mathrm{cm}^{-1}$ ) obtained with a Milli-Q reagent-grade water system (Millipore, Bedford, MA).

\section{Variable-pitch dispensing workstation}

Figure 1 is a schematic illustration of the 8-ch capillary-based dispensing workstation with a variable capillary pitch mechanism, developed based on recently submitted patents. ${ }^{11,12}$ This system consists of a dispensing head, a dispensing system, a microtiter plate holder, a work holder, and a wash space. Fused-silica capillaries (o.d. $360 \mu \mathrm{m}$ and i.d. $150 \mu \mathrm{m}$; Model FS-115, Upchurch Scientific, Oak Harbor, WA) are supported by arms, configuring a dispensing head. The arms are placed at even intervals by ball screws, the rotation of which can achieve the expansion and contraction of the capillary pitch in a range of 1 to $9 \mathrm{~mm}$. These 8 capillaries are translated with their ends resting on the same imaginary horizontal line at all times. Each capillary is connected to each syringe pump (Gastight \#1725, Hamilton, Reno, NV) through polyether ether ketone (PEEK) tubes, creating a dispensing system. The inside of the dispensing system is filled with water and reciprocation of the syringe plunger performs the aspiration and dispensing of sample solutions from the capillary end. The dispensing head can be freely moved both vertically and laterally between microtiter plates and work substrates or to the wash space, processing dispensing with or without contact of the capillary ends to the substrate surfaces. Work substrates such as glass slides or microsensor chips are attached to a work holder equipped with a vacuum chuck. The wash space is composed of Teflon reservoirs of water and ethanol and an $\mathrm{N}_{2}$ blow nozzle. After dispensing, the remaining sample solutions are pushed away with water filled inside the dispensing system. The capillaries are washed with water and ethanol by aspirating and dispensing them repeatedly, and then the remaining drops of the solvents are blown off from the capillaries by $\mathrm{N}_{2}$ gas flow. This dispensing workstation is connected to a laptop PC via a USB interface and can be controlled by dedicated software, by which the volumes, addresses, timing, speed, and other detailed information for aspirating and dispensing are easily configured.

\section{Evaluation of the dispensing precision}

An evaluation of the dispensing precision was performed as follows. A solution of Rhodamine $G$ dye was prepared by
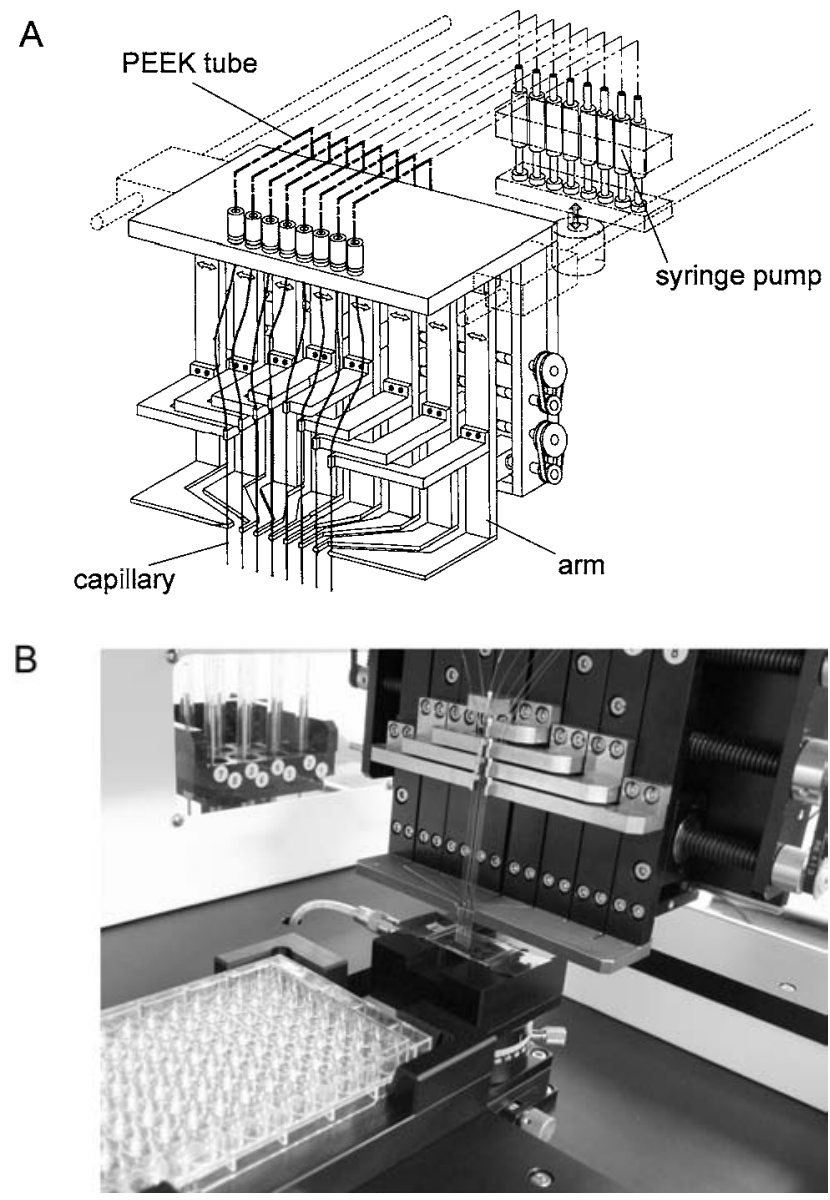

Fig. 1 Schematic illustration (A) and photograph (B) of the developed 8-ch capillary-based variable-pitch dispensing workstation.

adding $500 \mu \mathrm{L}$ of an $8 \mathrm{mM}$ Rhodamine $\mathrm{G}$ aqueous solution to $500 \mu \mathrm{L}$ of glycerol. Certain amounts of the solution were dispensed onto a glass slide (Nalge Nunc International, Tokyo, Japan). The diameters of dispensed droplets depend on the viscosity of the solutions and the wettability of the substrate surfaces; also, the minimum of the dispensing intervals depends on these factors as well as dispensing volumes. In this investigation, intervals of 1 or $2 \mathrm{~mm}$ were used for dispensing 20 and 50 or $100 \mathrm{~nL}$ of droplets, respectively, to prevent them from being mixed with adjacent droplets. The slide was scanned as a digital image using a GS-800 Calibrated Densitometer (BioRad Laboratories, Hercules, CA), and the image was processed using Quantity One image processing software (Bio-Rad Laboratories) to obtain values of the optical volume. Each value was calculated to be the sum of the intensities of the pixels (in optical density, OD) inside the spot boundary multiplied by the area of a single pixel (in $\mathrm{mm}^{2}$ ), corrected by subtracting the background. The measurement was performed over the range where the saturated pixels were not observed and the linearity was guaranteed.

\section{Preparation of microsensor array chips}

Microelectrode array chips (96 channels; pitch of $1 \mathrm{~mm}$; diameter of $c a .280 \mu \mathrm{m}$ (i.e., area of $0.062 \mathrm{~mm}^{2}$ )) were fabricated using a photolithographic strategy. A $15 \AA$ A-thick chromium adhesion layer and a $2000 \AA$-thick gold film were deposited on a mechanically polished barium borosilicate glass plate $(24 \times 36 \mathrm{~mm}, \# 1737$, Corning, NY) using a radio-frequency 

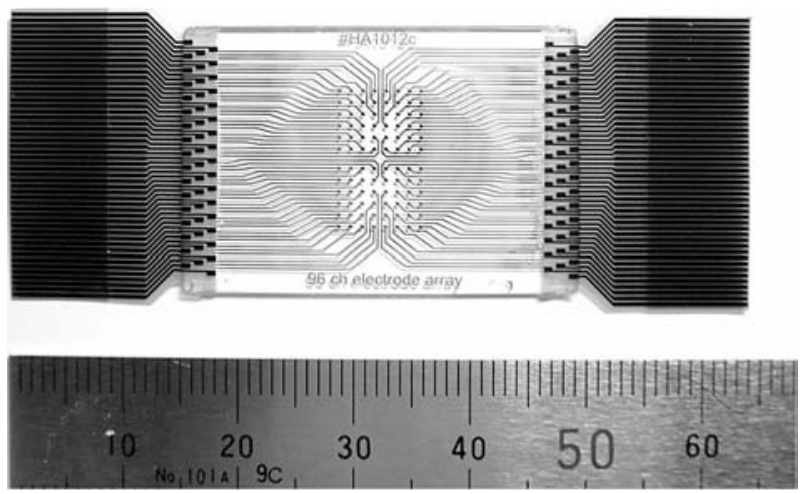

Fig. 2 Photograph of a fabricated 96-ch microelectrode array chip $(8 \times 12$ array, pitch of $1 \mathrm{~mm})$.

magnetron sputtering method employing a CFS-4EP-LL sputter equipment (Shibaura Mechatronics, Kanagawa, Japan). OMR 83 negative photoresist was spun on the plate for $30 \mathrm{~s}$ at $3000 \mathrm{rpm}$, exposed to UV irradiation using a Supercure-203S UV light source (San-ei electric, Osaka, Japan) for $8 \mathrm{~s}$ for patterning with the electrode array, and developed for $5 \mathrm{~min}$ in OMR Developer. The pre- and post-bakes were performed for $30 \mathrm{~min}$ at $85^{\circ} \mathrm{C}$ and at $120^{\circ} \mathrm{C}$, respectively. The excess metal was etched off by immersion in $\mathrm{KI} / \mathrm{I}_{2} /$ water $=4: 1: 40$ and $\mathrm{HClO}_{4} / \mathrm{Ce}\left(\mathrm{NH}_{4}\right)_{2}\left(\mathrm{NO}_{3}\right)_{6} /$ water $=5: 17: 100$ solutions for gold and chromium, respectively. The photoresist was removed by immersion in Remover 502A. SU-8 3000 was spun on the plate for $30 \mathrm{~s}$ at $2000 \mathrm{rpm}$, exposed for $8 \mathrm{~s}$ to cover the plate surface to restrict the electrode region, and developed for $5 \mathrm{~min}$ in SU-8 Developer. After developing, the plates were rinsed with 2-propanol. Both the pre- and post-bakes were performed for $1 \mathrm{~min}$ at $65^{\circ} \mathrm{C}+$ for $3 \mathrm{~min}$ at $95^{\circ} \mathrm{C}$. For signal output terminals, two flexible polyimide cables (50 pins; pitch of $0.5 \mathrm{~mm}$ ) were connected to both sides of the array chip with ACF by thermal compression bonding. The fabricated microelectrode array chip is shown in Fig. 2.

Microsensor array chips were prepared by modifying microelectrode surfaces with thiol derivatives, 11-HUT or 8-FcOT, by dispensing the corresponding solutions on the surfaces. The solutions of 11-HUT or 8-FcOT were prepared by adding $500 \mu \mathrm{L}$ of $1 \mathrm{mM} 11$-HUT or 8-FcOT ethanolic solutions to $500 \mu \mathrm{L}$ of a $50 \%$ glycerol aqueous solution, respectively. Fifty-nanoliter aliquots of the solutions were dispensed by the dispensing workstation on the microelectrode surfaces. After an 18-h immersion, the array chip was rinsed with ethanol and water, dried with an $\mathrm{N}_{2}$ blow, and stocked in a $0.1 \mathrm{M} \mathrm{NaClO}_{4}+$ $2.5 \mathrm{mM}$ phosphate buffer solution $\left(\mathrm{Na}^{+}\right.$salt, $\mathrm{pH}$ 7.0) until use.

\section{Topographical measurements}

Topographical measurements of the dimensions of the patterns fabricated on the plates were performed using an Alpha-Step IQ surface profiler (KLA-Tencor, San Jose, CA) and a VK-8500 color laser 3D profile microscope (Keyence, Osaka, Japan). The diameter of the microelectrode and the thickness of the SU-8 coating were observed to be $c a .280 \mu \mathrm{m}$ and $c a .10 \mu \mathrm{m}$, respectively.

\section{Electrochemical measurements}

Electrochemical measurements were performed at room temperature with a three-electrode configuration consisting of an $\mathrm{Ag} / \mathrm{AgCl}$ reference electrode, a platinum auxiliary electrode,
A

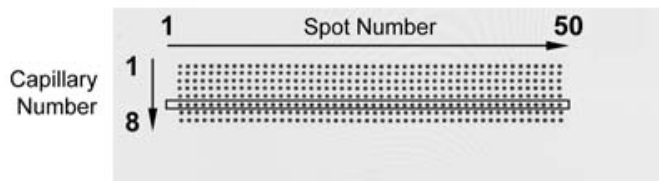

B

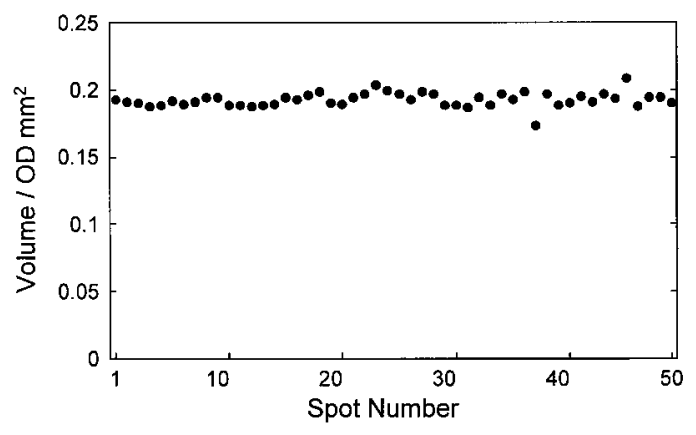

C

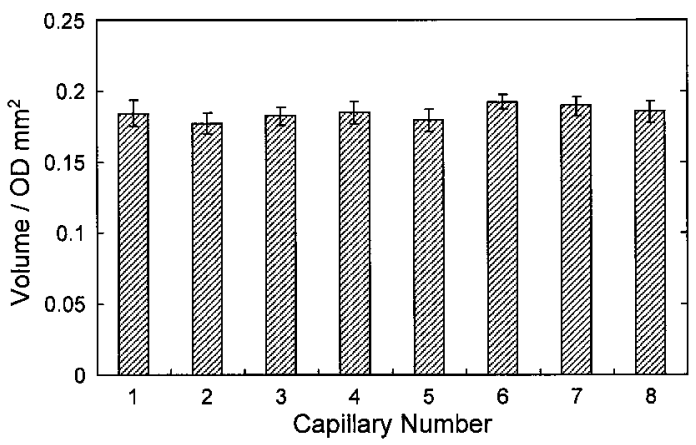

Fig. 3 (A) Scanned image of a glass slide, on which $4 \mathrm{mM}$ of Rhodamine $\mathrm{G}$ dye in $50 \%$ glycerol aqueous solution was dispensed in an $8 \times 50$ array of $20 \mathrm{~nL}$ spots at a pitch of $1 \mathrm{~mm}$. (B) Plots of the optical volumes versus spot numbers for 50 spots in a typical capillary number emphasized in Fig. 3A. (C) Comparison of the average values of the optical volume obtained for 8 capillaries $(n=50)$.

and working electrodes, using an HA-1010mM8S 8-ch potentiostat/galvanostat system (Hokuto Denko, Tokyo, Japan) combined with an NR-600 data logger system (Keyence) and a Model 2711A arbitrary waveform generator (Tegam, Geneva, $\mathrm{OH})$. The fabricated 96-ch microsensor array was connected as working electrodes to the potentiostat system. Electrochemical signals were recorded by every 8 microelectrodes in 12 separate measurements. Cyclic voltammetry was performed in a $0.1 \mathrm{M}$ $\mathrm{NaClO}_{4}+2.5 \mathrm{mM}$ phosphate buffer solution $\left(\mathrm{Na}^{+}\right.$salt, $\mathrm{pH}$ 7.0). The potential was scanned from -0.05 to $0.6 \mathrm{~V}$ and again back to $-0.05 \mathrm{~V}$ at a scan rate of $0.1 \mathrm{~V} \mathrm{~s}^{-1}$.

\section{Results and Discussion}

\section{Evaluation of dispensing precision}

Conventionally, the dispensed volume is evaluated by measuring the absorbance of a dispensed solution containing a known concentration of dye. If the dispensed volume is less than that needed for measurement, the solution is diluted until it reaches the requisite volume. Dilution of the solution, however, increases the measurement error, especially in the evaluation of smaller volumes, so another method is necessary to evaluate small-volume dispensing. Our system can dispense small volumes $(<10 \mu \mathrm{L})$ owing to its use of capillaries as dispenser heads. In this study, the precision of the dispensing performance 
Table 1 Values of the optical volume and the volume error when dispensing 20,50, and $100 \mathrm{~nL}$ of $4 \mathrm{mM}$ Rhodamine $\mathrm{G}$ dye in a $50 \%$ glycerol aqueous solution

\begin{tabular}{ccccc}
\hline $\begin{array}{c}\text { Dispensing } \\
\text { volume/nL }\end{array}$ & $\begin{array}{c}\text { Optical volume/ } \\
\text { OD mm }\end{array}$ & C.V., \% & $\begin{array}{c}\text { Volume error }{ }^{\mathrm{a}} / \\
\mathrm{nL}\end{array}$ & $n$ \\
\hline 20 & $0.192 \pm 0.00523$ & 2.7 & 0.54 & 50 \\
50 & $0.348 \pm 0.0107$ & 3.1 & 1.5 & 50 \\
100 & $0.603 \pm 0.0177$ & 2.9 & 2.9 & 25 \\
\hline
\end{tabular}

a. Volume error is a product of that of the dispensing volume and that of C.V.

was, therefore, evaluated based on direct observations of dispensed droplets on a glass slide by scanning the slide as a digital image to observe the spot volume.

Figure $3 \mathrm{~A}$ is a scanned image of a glass slide, on which a $50 \%$ glycerol aqueous solution containing $4 \mathrm{mM}$ of Rhodamine $\mathrm{G}$ dye was dispensed in an $8 \times 50$ array of $20 \mathrm{~nL}$ spots at a spot pitch of $1 \mathrm{~mm}$. This figure shows that the spot shape is close to circular; the diameter was calculated to be about $550 \mu \mathrm{m}$. The values of the optical volume for 50 spots in a typical capillary number are plotted versus the spot numbers in Fig. 3B, analyzed to be $0.192 \pm 0.00523 \mathrm{OD} \mathrm{m^{2 }}$ (a relative standard deviation of $2.7 \%$ ). The observed error in the spot volume was deduced from this relative error to be $0.54 \mathrm{~nL}(=2.7 \%$ of $20 \mathrm{~nL})$. The same evaluation was performed for an $8 \times 50$ array of $50 \mathrm{~nL}$ spots (diameter of about $690 \mu \mathrm{m}$ ) and an $8 \times 25$ array of $100 \mathrm{~nL}$ spots (diameter of about $910 \mu \mathrm{m}$ ), giving errors in the spot volume of $1.5 \mathrm{~nL}(=3.1 \%$ of $50 \mathrm{~nL})$ and $2.9 \mathrm{~nL}(=2.9 \%$ of 100 $\mathrm{nL})$, respectively, as summarized in Table 1. In this system 20 $\mathrm{nL}$ was the smallest volume for stable dispensing with the error in the spot volume of about $3 \%$, though smaller volumes could be dispensed with larger errors. In contrast, conventional dispensers using disposable pipette tips as dispensing heads generally have a relative standard deviation of about $5 \%$ at $1 \mu \mathrm{L}$ utilizing a $10-\mu \mathrm{L}$ tip: i.e., the observed error is about $50 \mathrm{~nL}$. Here, the volume of $1 \mu \mathrm{L}$ is so far the smallest practical volume for pipette dispensing. Therefore, in dispensing small volumes, the error in the dispensed spot volume for this dispenser was found to be about 100-fold less than that seen in conventional dispensers.

We believe that the high precision of this dispenser has been achieved by connecting capillaries to syringe pumps through PEEK tubes, which have the same ability to withstand high pressure as stainless-steel tubes. Also, by filling the inside with water, an incompressible fluid, instead of air, which is compressive, contributed to this performance by eliminating the damper effect from the presence of air. It should be noted that a gap was made with quite a small amount $(\sim 0.5 \mu \mathrm{L})$ of air between the water and the sample solution to prevent any admixture. Despite this, the highly viscous sample solution prepared with $50 \%$ glycerol aqueous solution could be dispensed with a high degree of precision, giving evidence that this dispenser can be applied to various kinds of solutions including biological samples with high viscosity.

Figure $3 \mathrm{C}$ shows a comparison of the average values of the optical volume obtained for 8 capillaries. The values for the droplets dispensed from the 8 capillaries were the same within the standard deviation. This result shows the reproducible dispensing ability of this dispensing workstation for simultaneous multiple spotting.

Simultaneous dispensing of 8 spots was completed in $34 \mathrm{~s}$ : that is the dispensing time of $4.3 \mathrm{~s}$ per one spot. Compared to

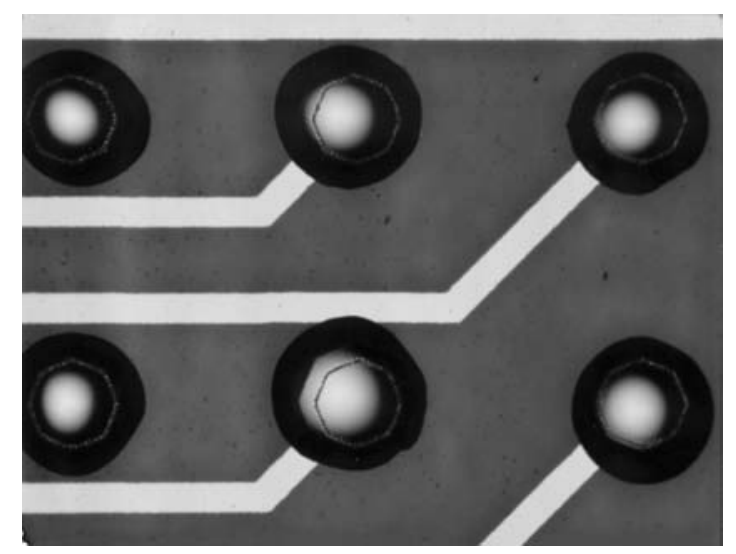

Fig. 4 Photograph of microelectrodes loaded with solution droplets dispensed by the developed dispensing workstation.

this, conventional pipette-based dispensers need about 30 - 60 min for dispensing 96 spots of different solutions with the same spot interval of $1 \mathrm{~mm}$ : that is the dispensing time of $18.8-37.5$ $\mathrm{s}$ per one spot. From these facts, dispensing with this workstation was found to be 4.4 - 8.7-fold faster than that with conventional dispensers. This ability of rapid dispensing was realized by using a variable-pitch multi-channel dispensing head enabling us to dispense simultaneously different solutions directly to substrates.

\section{Microsensor array chips}

Preparing independently addressable microsensors on chips is the key to the simultaneous detection of multiple components including gene analysis based on arrays of high-density fabricated microelectrodes. Moreover, surface modification of gold-thin-film-based microelectrodes requires the rapid dispensing of small volumes of solutions to ensure monolayer uniformity. As described in the previous section, the developed dispensing workstation can simultaneously dispense 8 small droplets of solutions at narrow pitches. The fabrication of a microsensor array was therefore demonstrated in this section as a potential application of the dispensing workstation using model thiols, electroinactive 11-HUT and electroactive 8-FcOT.

Solutions of 11-HUT and 8-FcOT were dispensed on a photolithographically fabricated $8 \times 12$ microelectrode array chip, with 11-HUT and 8-FcOT solutions dispensed on microelectrodes in odd and even rows, respectively. The solutions included $25 \%$ glycerol to prevent the dispensed solutions from drying during monolayer formation. Figure 4 shows the microelectrodes loaded with solution droplets that had not mixed with adjacent droplets. Cyclic voltammograms for the thus-prepared microsensor array chip were electrochemically measured in a $0.1 \mathrm{M} \mathrm{NaClO}_{4}+2.5 \mathrm{mM}$ phosphate buffer $\left(\mathrm{Na}^{+}, \mathrm{pH}\right.$ 7.0) solution. Figure 5 shows cyclic voltammograms for microsensors in a typical column of the array. All of the microsensors in the even rows showed anodic and cathodic peaks attributable to ferrocene redox reactions around $0.3 \mathrm{~V}$ vs. $\mathrm{Ag} / \mathrm{AgCl}$, while those in the odd rows showed only charging currents. This result shows that 11-HUT and 8-FcOT clearly did not mix with each other on the chip and that they were independently and reproducibly immobilized on microelectrodes at an interval of $1 \mathrm{~mm}$. An addressable microsensor array was thus achieved. From this result, we conclude that the dispensing workstation can dispense solutions at narrow pitches not only on glass slides with ideally prepared 


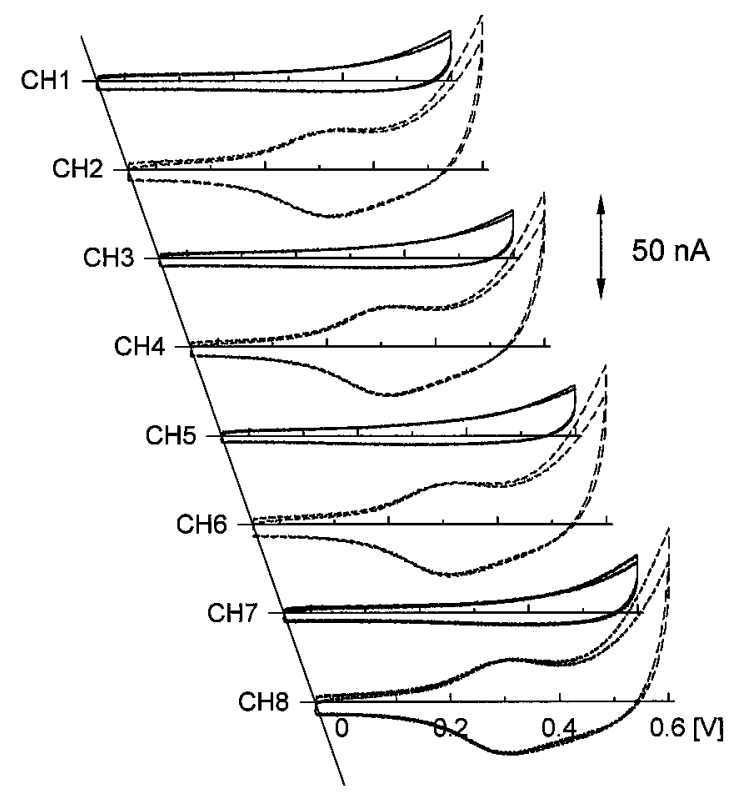

Fig. 5 Cyclic voltammograms for microsensors in a typical column of the prepared array. Microelectrodes in the odd rows $(\mathrm{CH} 1,3,5$, and 7, solid line) were modified with 11-HUT and those in the even rows $(\mathrm{CH} 2,4,6$, and 8, dashed line) were modified with 8-FcOT.

surfaces but also on freely fabricated microelectrode sensor chips, providing an independent and reproducible spot array.

\section{Conclusion}

In this study, we developed a dispensing workstation installed with a variable capillary pitch mechanism, with a dispensing pitch that is able to expand or contract in the range from 1 to 9 $\mathrm{mm}$. The variable-pitch mechanism makes it possible to dispense different solutions simultaneously and directly from microplates to integrated analytical systems. By dispensing droplets on glass slides, this workstation was found to have a volume error of $0.54 \mathrm{~nL}$ when dispensing $20 \mathrm{~nL}$, indicating much more precise dispensing than that seen in conventional dispensers that use disposable pipette tips. The dispensing proved to be 4.4-8.7-fold faster compared to conventional dispensers. Moreover, the narrow-pitch dispensing of different solutions was demonstrated by using this workstation to prepare addressable microsensor array chips, providing evidence for the reproducible performance of the workstation.

This workstation can dispense highly viscous solutions such as biologically relevant materials with rapidity, high precision, and high density. This fact demonstrates its potential application to high-throughput analysis including mass spectrometry. It also has potential for nanobiotechnological applications to nanobiodevices or array-based analysis, e.g., microchips and protein or cell array chips, since the workstation allows the simultaneous positioning of different sample droplets with high density, which is not possible with conventional dispensing systems.

\section{Acknowledgements}

The authors are grateful to M. Arifuku and N. Mochizuki of Hitachi Chemicals for their technical support with thermal compression bonding. This work was partially supported by a Grant-in-Aid for Young Scientists (B) from the Ministry of Education, Culture, Sports, Science and Technology, No. 19750068.

\section{References}

1. P. Bevan, H. Ryder, and I. Shaw, Trends Biotechnol., 1995, 13,115 .

2. A. Smith, Nature, 2002, 418, 453.

3. G. L. Hortin, Clin. Chem., 2006, 52, 1223.

4. C. Pan, S. Xu, H. Zhou, Y. Fu, M. Ye, and H. Zou, Anal. Bioanal. Chem., 2007, 387, 193.

5. M. J. Heller, Annu. Rev. Biomed. Eng., 2002, 4, 129.

6. E. Phizicky, P. I. H. Bastiaens, H. Zhu, M. Snyder, and S. Fields, Nature, 2003, 422, 208.

7. J. Ziauddin and D. M. Sabatini, Nature, 2001, 411, 107.

8. T. Yoshikawa, E. Uchimura, M. Kishi, D. P. Funeriu, M. Miyake, and J. Miyake, J. Controlled Release, 2004, 96, 227.

9. S. N. Bailey, S. M. Ali, A. E. Carpenter, C. O. Higgins, and D. M. Sabatini, Nature Methods, 2006, 3, 117.

10. T. Chapman, Nature, 2003, 421, 661.

11. H. Aoki, M. Torimura, H. Tao, and T. Ikeda, International Patent Publication, No. WO/2008/007556.

12. H. Aoki, M. Torimura, H. Tao, and T. Ikeda, Japanese Patent Application, No. 2008-004196. 